\title{
Menstrual hygiene practices and school absenteeism among adolescent girls in Bangladesh: A cross-sectional study
}

\author{
Mehedi Hasan ${ }^{1}$, Md Nazmul Hassan ${ }^{1}$, Mohsina H. Mita², Fatema T. Zahara², Md Hasib ${ }^{1}$
}

\author{
AFFILIATION \\ 1 Department of Environmental Sanitation, Patuakhali Science and \\ Technology University, Patuakhali, Bangladesh \\ 2 Faculty of Nutrition and Food Science, Patuakhali Science and \\ Technology University, Patuakhali, Bangladesh
}

\section{CORRESPONDENCE TO}

Mehedi Hasan. Department of Environmental Sanitation, Patuakhali Science and Technology University, Patuakhali - 8602, Bangladesh.

Popul. Med. 2021;3(March):8
E-mail: mehedi.nfs.pstu@gmail.com ORCID ID: https://orcid.org/00000002-4974-4057

\section{KEYWORDS}

Bangladesh, school-going adolescent girls, school absenteeism, menstrual hygiene practices

Received: 7 September 2020, Revised: 23 October 2020,

Accepted: 22 February 2021

https://doi.org/10.18332/popmed/133641

\begin{abstract}
INTRODUCTION Menstrual hygiene practices refers to maintaining specific hygiene during menstrual periods. Excused or unexcused school absenteeism can be attributed to adopted menstrual hygiene practices. This study aimed to assess the prevalence of school absenteeism during menstrual cycle and to explore the association of menstrual hygiene practices with school absenteeism among schoolgoing adolescent girls in Bangladesh.

METHODS A cross-sectional study was conducted from April to November 2019 based on a sample of 442 school-going adolescent girls (aged 10-19 years) from randomly selected nine Girls' Schools in the Dhaka division, Bangladesh. Data were collected by an interviewer-administered questionnaire and a multiple regression model was applied to assess factors associated with school absenteeism.

RESULTS The prevalence of school absenteeism was (35.1\%) among school-going adolescent girls in Bangladesh.
\end{abstract}

Factors associated with school absenteeism included older adolescent girls (AOR=2.97; 95\% CI: 1.66-5.24, $\mathrm{p}<0.05$ ), with an illiterate mother (AOR=5.36; 95\% CI: 1.91-12.44, $\mathrm{p}<0.05)$ and illiterate father (AOR $=4.66$; $95 \% \mathrm{CI}: 1.79-11.24$, $\mathrm{p}<0.001$ ), from single families (AOR $=2.54 ; 95 \%$ CI: $1.45-4.47$, $\mathrm{p}<0.001$ ), who did not know about the menstruation cycle before menarche $(\mathrm{AOR}=2.14 ; 95 \% \mathrm{CI}: 1.32-3.48, \mathrm{p}<0.001)$ and who practised poor hygiene management $(\mathrm{AOR}=5.66$; 95\% CI: 2.73-15.32, p<0.001). Poor hygiene management including the lack of sanitary pads, the washing of a reusable cloth without soap/antiseptics, and lack of bathing and cleaning external genitalia, were factors associated with school absenteeism.

CONCLUSIONS The study findings may help governmental and non-governmental organizations design interventions to improve knowledge on the menstrual cycle and so reduce school absenteeism during menstrual periods.

\section{INTRODUCTION}

Menstrual hygiene practices refer to maintaining a specific hygiene by women and adolescent girls during the menstruation cycle, including sanitary material to absorb or collect menstrual blood and facilities for disposing them, in order to effectively manage the menstrual period ${ }^{1,2}$. Adolescent girls from low- and middle-income countries face different challenges to maintain proper hygiene during menstruation ${ }^{3}$. Low quality sanitary materials, the social culture related to menstruation hygiene management, inadequate water, sanitation and hygiene (wash) facilities, lack of guidance and privacy for changing and washing at school or home are common issues related to inadequate menstrual hygiene practices that have adverse effects on education, health and economic outcomes ${ }^{4}$.

School absenteeism means missing school due to excused or unexcused reasons. School absenteeism may also occur during the menstrual cycle due to poor menstrual hygiene practices attributed to the improper school setting, lack of gender-specific wash facilities, insufficient sanitary material, 
and bad response/attitude by male teachers and students, etc. 5,6 Menstrual fatigue and pain are also responsible for school absenteeism. As a result, reduced school performance, drop-out from school, and lower educational attainment appear to have long-term effects on gender equality, education, sexual and reproductive health, and economic outcomes ${ }^{6,7}$.

In Bangladesh, adolescent girls have negative attitudes towards menstrual hygiene practices. Many adolescent girls lack scientific knowledge about the menstrual cycle. They feel hesitant to discuss menstruation with their parents, relatives, friends and others ${ }^{8}$. The majority of adolescent girls in Bangladesh do not know about menarche or maintain menstrual hygiene. The Bangladesh Demographic Health Survey 2014 reported that only $33.7 \%$ of adolescent girls (aged 15-19 years) had heard about menstruation before menarche9. A study conducted among adolescent girls in a rural area in Bangladesh reported that about $69 \%$ used an unhygienic cloth or even no protection during menstruation. Only about $24 \%$ of school settings fulfil basic wash facility criteria, which is a challenge to improve these facilities in Bangladesh ${ }^{10}$. According to the National Baseline Survey 2014 in Bangladesh, there is on average 1 toilet per 187 students with insufficient water and soap facilities in nearly two-thirds of the bathrooms. In addition, there is a lack of light and ventilation facilities ${ }^{11}$.

Knowledge, attitudes, and practices on menstrual hygiene management and its impact among Bangladeshi adolescent girls are not well known, with little evidencebased intervention programs to reduce school absenteeism among adolescent girls during menstrual periods. To address this information gap, a cross-sectional study was conducted among Bangladeshi adolescent girls aimed to assess the prevalence of school absenteeism during menstrual periods and to explore the association of menstrual hygiene practices with school absenteeism, which can help governmental and non-governmental organizations to design programs to reduce school absenteeism and so improve the educational status of women.

\section{METHODS}

\section{Study settings}

The study was conducted from April to November 2019 among school-going adolescent girls in three rural areas of Bangladesh (Dhaka, Manikgonj and Gazipur). These three districts were randomly selected from the Dhaka Division in Bangladesh. We collected data from three different Girls' Schools from each district.

\section{Study design}

This cross-sectional study was conducted among schoolgoing adolescent girls present in the school during the data collection process. We invited all students to participate in the study and informed them about the purpose of the study, and ensured that the study had no personal implications. We only considered grades 7 to 10 because most of the girls in this class represent adolescent girls (aged 10-19 years) who reached menarche.

\section{Sample size estimation and procedure}

The minimum required sample size was calculated by using a single population proportion formula $\left(\mathrm{n}_{\mathrm{o}}=\mathrm{Z}^{2} \mathrm{p} \mathrm{q} / \mathrm{d}^{2}\right), 5 \%$ margin of error, $95 \%$ confidence intervals, and an estimated prevalence of school absenteeism of $40 \%$, based on schoolgoing adolescent girls during the menstrual cycle in India (developing country like Bangladesh) ${ }^{12}$. This yielded a sample size of 442 with $20 \%$ non-response rate. A simple random sampling process was used for this study. At first, we randomly selected girls' schools from the list of the district education office for each district and then randomly selected participants from the attendance list of the respective classes. If the chosen girl was not interested in the study, she was excluded.

\section{Study variables}

Outcome variables were school absenteeism during menstruation and poor menstrual hygiene practices. School absenteeism was defined as having missed school during the menstrual cycle. According to a study measure developed by WaterAid and DFID ${ }^{13}$, menstrual hygiene practices are considered as poor when girls report any one of the following behaviors: changing MHM (menstruation hygiene management) material less frequently than every 8 hours; not cleaning hands before and after changing MHM materials; not washing external genitalia at least once per day; and using reusable sanitary pads/cloths without washing them with soap/antiseptic.

Independent variables included sociodemographic (age, parents' education and occupation, type of family, religion, family income, etc.) and menstrual hygiene practices information.

\section{Data collection}

A female research assistant was recruited and trained for fieldwork, which included sample selection and collection of data by reviewing the questionnaire through teamwork with the researcher. Structured questionnaires were used to collect data. A random sampling approach was adopted to collect information like menstrual hygiene practices, the menstrual cycle pattern, and factors associated with school absenteeism through face-to-face interviews. The protocol of this study was approved by the Research Ethical Committee (REC) of the Department of Environmental Sanitation, Patuakhali Science and Technology University, Bangladesh (approval no. ENS: 26/02/2019:04). The purpose of the study was explained in detail to the participants, and written informed consent was obtained from the subjects before participation in the study. Moreover, permission was obtained from the principals of the schools. 


\section{Statistical analysis}

The quantitative data were analyzed using SPSS for Windows Version 23.0. Descriptive statistics, such as frequency, percentages, mean and standard deviation, were used to analyze the demographic details of the respondents. Multiple logistic regression analyses were performed to utilize factors associated with poor menstrual hygiene practices and school absenteeism during the menstruation cycle. Hosmer and Lemeshow test was used to express the goodness-of-fit of the multiple regression model. The adjusted odds ratio (AOR) was used to evaluate the strength of associated factors with poor menstrual hygiene practices and school absenteeism during the menstruation cycle at $95 \% \mathrm{CI}$, and $\mathrm{p}<0.05$ was considered statistically significant.

\section{RESULTS}

Sociodemographic characteristics of school-going adolescent girls in Bangladesh are shown in Table 1. Of 442 participants, about one-third $(35.1 \%)$ of adolescent girls were absent from school, and more than half (62.3\%) of the adolescent girls were aged $\geq 15$ years, with a mean $( \pm S D)$ age of 16.8 $( \pm 5.3)$ years. Most of the respondents $(94.3 \%)$ were Muslims. About half of the respondents' mothers (54.1\%) had secondary or above education level, and for the majority of $(74.8 \%)$ respondents, their mother was a housewife. Moreover, about half of the respondents' fathers were employed (53.4\%) and had secondary or above education level (52.3\%). About $43.2 \%$ of respondents did not know about the menstruation cycle before menarche; more than half $(54.9 \%)$ of the respondents knew from their mother and $12.4 \%$ from a school-based program. The majority (67.2\%) of the respondents did not know the reason for menstruation, only $30.3 \%$ of respondents knew that it is a physiological process, and $2.9 \%$ believed that it is a curse of God.

Table 2 shows the bivariate regression analysis used to identify sociodemographic factors associated with school absenteeism among school-going adolescent girls. The Hosmer and Lemeshow test $\left(\chi^{2}=8, d f=6, p=0.76\right)$ was used to express the goodness-of-fit. The adjusted regression model showed that the following sociodemographic factors were statistically significant with school absenteeism among adolescent girls: older age (AOR=2.97; 95\% CI: 1.66-5.24, $\mathrm{p}<0.05$ ); illiterate mother (AOR $=5.36$; $95 \% \mathrm{CI}: 1.91-12.44$, $\mathrm{p}<0.05)$ and illiterate father $(\mathrm{AOR}=4.66$; $95 \% \mathrm{CI}: 1.79-11.24$, $\mathrm{p}<0.001$ ); from single families (AOR $=2.54$; $95 \%$ CI: $1.45-4.47$, $\mathrm{p}<0.001$ ); did not know about the menstruation cycle before menarche (AOR=2.14; 95\% CI: $1.32-3.48, \mathrm{p}<0.001)$; and poor hygiene management (AOR=5.66; 95\% CI: 2.73-15.32, $\mathrm{p}<0.001$ ). Moreover, school absenteeism was less frequent among adolescent girls who received information about the menstrual cycle from school-based programs $(\mathrm{AOR}=0.17$;

Table 1. Sociodemographic characteristics of school-going adolescent girls in Bangladesh $(\mathrm{N}=442)$

\begin{tabular}{|c|c|c|c|c|}
\hline \multirow[t]{2}{*}{ Characteristics } & \multirow{2}{*}{$\begin{array}{l}\text { Total } \\
\text { n (\%) }\end{array}$} & \multicolumn{2}{|c|}{ Absenteeism } & \multirow[b]{2}{*}{$\mathbf{p}$} \\
\hline & & $\begin{array}{c}\text { Yes } \\
\text { n (\%) }\end{array}$ & $\begin{array}{c}\text { No } \\
\text { n (\%) }\end{array}$ & \\
\hline Total & & $155(35.1)$ & $287(64.9)$ & \\
\hline \multicolumn{5}{|l|}{ Age (years) } \\
\hline$<15$ & $167(37.8)$ & $27(16.2)$ & $140(83.8)$ & \\
\hline$\geq 15$ & $275(62.2)$ & $127(46.2)$ & $148(53.8)$ & $0.047^{a}$ \\
\hline \multicolumn{5}{|l|}{ Religion } \\
\hline Muslim & 407 (94.3) & $128(31.5)$ & $279(68.5)$ & 0.167 \\
\hline Non-Muslim & 35 (5.7) & 27 (77.1) & $8(22.9)$ & \\
\hline \multicolumn{5}{|c|}{ Mother's education level } \\
\hline Illiterate & $75(16.9)$ & $53(70.7)$ & $22(29.3)$ & \\
\hline Primary & 128 (28.9) & $55(43.0)$ & $73(57.0)$ & $0.035^{a}$ \\
\hline Secondary or above & $239(54.2)$ & 47 (19.7) & $192(80.3)$ & \\
\hline \multicolumn{5}{|l|}{ Mother's occupation } \\
\hline Housewife & 331 (74.9) & $79(24.0)$ & $252(76.0)$ & $0.004^{a}$ \\
\hline Employed or other & $111(25.1)$ & $76(68.5)$ & $35(31.5)$ & \\
\hline \multicolumn{5}{|c|}{ Father's education level } \\
\hline Illiterate & $58(13.1)$ & $39(67.2)$ & 19 (32.8) & \\
\hline Primary & $153(34.6)$ & $64(41.8)$ & 89 (58.2) & $0.007^{a}$ \\
\hline Secondary or above & $231(52.3)$ & $52(22.5)$ & $179(77.5)$ & \\
\hline
\end{tabular}


Table 1. Continued

\begin{tabular}{|c|c|c|c|c|}
\hline \multirow[t]{2}{*}{ Characteristics } & \multirow{2}{*}{$\begin{array}{l}\text { Total } \\
\text { n (\%) }\end{array}$} & \multicolumn{2}{|c|}{ Absenteeism } & \multirow[b]{2}{*}{$\mathbf{p}$} \\
\hline & & $\begin{array}{c}\text { Yes } \\
\text { n (\%) }\end{array}$ & $\begin{array}{c}\text { No } \\
\text { n (\%) }\end{array}$ & \\
\hline \multicolumn{5}{|l|}{ Father's occupation } \\
\hline Farmer & $189(42.8)$ & $47(24.9)$ & $142(75.1)$ & $0.046^{\mathrm{a}}$ \\
\hline Employed or other & $253(57.2)$ & $108(42.7)$ & $189(57.3)$ & \\
\hline \multicolumn{5}{|l|}{ Type of family } \\
\hline Extended & $275(62.2)$ & $66(24.0)$ & $209(76.0)$ & $0.008^{\mathrm{a}}$ \\
\hline Single/nuclear & $167(37.8)$ & $89(53.3)$ & $78(46.7)$ & \\
\hline \multicolumn{5}{|c|}{ Knew about menstruation cycle before menarche } \\
\hline Yes & $251(56.8)$ & $53(21.1)$ & $198(78.9)$ & $0.002^{b}$ \\
\hline No & $191(43.2)$ & $102(53.4)$ & $89(46.6)$ & \\
\hline \multicolumn{5}{|c|}{ Source of information before menarche } \\
\hline Mother & $243(55.0)$ & $80(32.9)$ & $163(67.1)$ & \\
\hline Relatives/friends & $78(17.6)$ & $37(47.4)$ & $41(52.6)$ & $0.035^{\mathrm{a}}$ \\
\hline TV/movies & $66(14.9)$ & $25(37.9)$ & $41(62.1)$ & \\
\hline School program & $55(12.5)$ & $13(23.6)$ & $42(76.4)$ & \\
\hline \multicolumn{5}{|c|}{ Thoughts about menstruation } \\
\hline Don't know & $297(67.2)$ & $86(29.0)$ & $211(71.0)$ & \\
\hline Physiological process & $134(30.3)$ & $62(46.3)$ & $72(53.7)$ & $0.751^{\mathrm{a}}$ \\
\hline Curse of God & $11(2.5)$ & $7(63.6)$ & $04(36.4)$ & \\
\hline
\end{tabular}

a Chi-squared test. b Fisher's exact test. Bold values are statistically significant.

Table 2. Factors associated with school absenteeism among school-going adolescent girls in Bangladesh

\begin{tabular}{|c|c|c|c|}
\hline Characteristics & OR $(95 \% \mathrm{CI})$ & AOR (95\% CI) & $\mathbf{p}$ \\
\hline \multicolumn{4}{|l|}{ Age (years) } \\
\hline$<15$ & 1.0 & 1.0 & \\
\hline$\geq 15$ & $3.53(1.98-5.25)^{* * *}$ & $2.97(1.66-5.24)$ & 0.026 \\
\hline \multicolumn{4}{|l|}{ Religion } \\
\hline Muslim & 1.0 & 1.0 & \\
\hline Non-Muslim & $1.47(1.01-2.88)^{*}$ & $0.81(0.37-1.48)$ & 0.526 \\
\hline \multicolumn{4}{|c|}{ Mother's education level } \\
\hline Illiterate & $8.27(3.53-17.74)^{* * *}$ & $5.36(1.91-12.44)$ & 0.016 \\
\hline Primary & $4.31(2.12-9.02)^{* * *}$ & $3.11(1.37-7.04)$ & 0.025 \\
\hline Secondary or above & 1.0 & 1.0 & \\
\hline \multicolumn{4}{|l|}{ Mother's occupation } \\
\hline House wife & 1.0 & 1.0 & \\
\hline Employed & $2.87(1.83-4.51)^{* * *}$ & $2.42(1.39-4.23)$ & 0.002 \\
\hline \multicolumn{4}{|c|}{ Father's education level } \\
\hline Illiterate & $7.91(2.53-15.84)^{* * *}$ & $4.66(1.79-11.24)$ & 0.004 \\
\hline Primary & $4.37(2.17-8.27)^{* * *}$ & $2.05(1.17-6.04)$ & 0.037 \\
\hline Secondary or above & 1.0 & 1.0 & \\
\hline
\end{tabular}


Table 2. Continued

\begin{tabular}{|c|c|c|c|}
\hline Characteristics & OR $(95 \% \mathrm{CI})$ & AOR $(95 \% \mathrm{CI})$ & $\mathbf{p}$ \\
\hline \multicolumn{4}{|c|}{ Father's occupation } \\
\hline Farmer & 1.0 & 1.0 & \\
\hline Employed & $1.93(1.05-3.14)^{* *}$ & $1.54(0.69-2.47)$ & 0.486 \\
\hline \multicolumn{4}{|l|}{ Type of family } \\
\hline Extended & 1.0 & 1.0 & \\
\hline Single/nuclear & $2.98(1.99-4.45)^{* * *}$ & $2.54(1.45-4.47)$ & 0.003 \\
\hline \multicolumn{4}{|c|}{ Knew about menstruation cycle before menarche } \\
\hline Yes & 1.0 & 1.0 & \\
\hline No & $3.25(2.23-4.74)^{* * *}$ & $2.14(1.32-3.48)$ & 0.002 \\
\hline \multicolumn{4}{|c|}{ Source of information before menarche } \\
\hline Mother & 1.0 & 1.0 & \\
\hline Relatives/friends & $1.57(1.21-3.81)^{* * *}$ & $1.37(1.04-2.69)$ & 0.039 \\
\hline TV/movies & $1.86(1.26-4.89)^{* * *}$ & $1.44(1.15-2.79)$ & 0.004 \\
\hline School program & $0.24(0.09-0.81)^{* * *}$ & $0.17(0.04-0.69)$ & 0.005 \\
\hline \multicolumn{4}{|c|}{ Menstrual hygiene management } \\
\hline Good & 1.0 & 1.0 & \\
\hline Poor $^{\mathrm{a}}$ & $8.91(3.86-19.81)^{* * *}$ & $5.66(2.73-15.32)$ & 0.008 \\
\hline
\end{tabular}

OR: odds ratio. AOR: adjusted odds ratio. CI: confidence interval. ${ }^{*} \mathrm{p}<0.05,{ }^{* *} \mathrm{p}<0.01,{ }^{* * *} \mathrm{p}<0.001$. Bold values indicate statistically significant. Multiple logistic model fit by Hosmer and Lemeshow test $\left(\chi^{2}=8, d f=6, p=0.76\right)$. a Less frequently than every 8 hours; not cleaning hands before and after changing materials; not washing external genitalia at least once per day; using reusable sanitary pad/cloth but not washing it with soap/antiseptic.

95\% CI: 0.04-0.69, p<0.001).

Table 3 depicts the findings of multiple logistic regression analysis used to identify the association of menstrual hygiene practices with school absenteeism among schoolgoing adolescent girls. The adjusted regression analysis revealed that the following menstrual hygiene practices were significant with school absenteeism: use of a homemade cloth for sanitary protection (AOR=2.14; 95\% CI: 1.23-5.79, p<0.01); underwear instead of a sanitary pad $(\mathrm{AOR}=2.54 ; 95 \% \mathrm{CI}: 1.43-2.82, \mathrm{p}<0.01)$; reuse of sanitary material (AOR=1.74; 95\% CI: 1.21-2.31, p<0.005); washing reusable cloth with only water $(\mathrm{AOR}=3.78$; $95 \% \mathrm{CI}: 1.27-$

Table 3. The association of menstrual hygiene practices with school absenteeism among school-going adolescent girls in Bangladesh $(\mathrm{N}=155)$

\begin{tabular}{|c|c|c|c|}
\hline \multirow[t]{2}{*}{ Characteristics } & Absenteeism & AOR $(95 \% \mathrm{CI})$ & $\mathbf{p}$ \\
\hline & $\begin{array}{c}\text { Yes } \\
\text { n (\%) }\end{array}$ & & \\
\hline \multicolumn{4}{|l|}{ Types of sanitary protection } \\
\hline Sanitary pad (Ref.) & $61(39.3)$ & - & \\
\hline Homemade cloth & $68(43.9)$ & $2.14(1.23-5.79)$ & 0.031 \\
\hline Underwear & $26(16.8)$ & $2.54(1.43-2.82)$ & 0.042 \\
\hline \multicolumn{4}{|l|}{ Dispose or reuse } \\
\hline Dispose (Ref.) & $61(39.4)$ & - & \\
\hline Reuse & $94(60.6)$ & $1.74(1.21-2.31)$ & 0.007 \\
\hline \multicolumn{4}{|l|}{ Washing of reusable cloth } \\
\hline Only water & $29(18.7)$ & $3.78(1.27-11.14)$ & 0.004 \\
\hline Water and soap & $86(55.5)$ & $1.54(1.03-2.11)$ & 0.031 \\
\hline Water with antiseptic (Ref.) & $40(25.8)$ & - & \\
\hline
\end{tabular}


Table 3. Continued

\begin{tabular}{|c|c|c|c|}
\hline \multirow[t]{2}{*}{ Characteristics } & Absenteeism & AOR $(95 \% \mathrm{CI})$ & $\mathbf{p}$ \\
\hline & $\begin{array}{c}\text { Yes } \\
\text { n (\%) }\end{array}$ & & \\
\hline \multicolumn{4}{|l|}{ Place of drying reusable cloth } \\
\hline Outside house in sunlight (Ref.) & $12(7.7)$ & - & \\
\hline Inside house & $58(37.5)$ & $1.98(1.04-3.89)$ & 0.425 \\
\hline Outside house without sunlight & $85(54.8)$ & $1.23(1.01-2.96)$ & 0.235 \\
\hline \multicolumn{4}{|l|}{ Bathing in this period } \\
\hline Only water & $98(63.2)$ & $1.93(1.09-3.69)$ & 0.002 \\
\hline Soap with water (Ref.) & $57(36.8)$ & - & \\
\hline \multicolumn{4}{|l|}{ Cleaning of external genitalia } \\
\hline Satisfactory (Ref.) ${ }^{\mathrm{a}}$ & $80(15.5)$ & & \\
\hline Unsatisfactory $^{\mathrm{b}}$ & $75(84.5)$ & $4.37(1.19-7.54)$ & 0.008 \\
\hline \multicolumn{4}{|c|}{ Material used for cleaning external genitalia } \\
\hline Only water & $132(85.0)$ & $9.87(2.17-15.69)$ & 0.021 \\
\hline Soap with water & $17(11.0)$ & $1.31(0.94-2.85)$ & 0.067 \\
\hline Water and antiseptic (Ref.) & $6(4.0)$ & - & \\
\hline \multicolumn{4}{|c|}{ Sanitary pads/cloths changed per day } \\
\hline 1 & $75(48.4)$ & $3.14(1.36-7.45)$ & 0.036 \\
\hline $2-3$ & $69(44.5)$ & $2.35(1.01-3.52)$ & 0.007 \\
\hline 3-4 (Ref.) & $11(7.1)$ & - & \\
\hline
\end{tabular}

AOR: adjusted odds ratio. CI: confidence interval. Ref.: reference. a Cleaning of external genitalia more than two times per day during menstruation. b Cleaning of external genitalia less than or equal to two times per day during menstruation. Bold values indicate statistically significant. Multiple logistic model fit by Hosmer and Lemeshow test $\left(\chi^{2}=9, \mathrm{df}=8, \mathrm{p}=0.68\right)$.

$11.14, \mathrm{p}<0.001)$; bathing using only water in this period (AOR=1.93; 95\% CI: 1.09-3.69, p<0.001); unsatisfactory cleaning of external genitalia (AOR=4.37; 95\% CI: 1.19-7.54, $\mathrm{p}<0.005$ ); and sanitary pad/cloth changed once per day (AOR=3.14; 95\% CI: 1.36-7.45, p<0.01).

\section{DISCUSSION}

We found that about one-third (35.1\%) of school-going adolescent girls were absent from school during menstrual periods, which is in accordance with a study conducted among adolescent girls in India $(40 \%)^{12}$. Another study conducted in Nepal reported that girls were more likely to be absent from school during menstruation than on other days ${ }^{14}$. This study identified that the older adolescent girls, whose mother was illiterate, and came from a nuclear family were significantly associated with higher school absenteeism during menstrual periods. Older adolescent girls were more absent from school compared with younger girls, and this was consistent with previous studies ${ }^{4,15}$. Overall, school absenteeism was higher among the older age group, indicating that age may be a significant factor associated with school absenteeism. School absenteeism was higher among the adolescent girls whose parents were illiterate, a result also found in a study conducted among Nigerian school-going girls ${ }^{16}$. Literate mothers play an important role in motivating their daughters regarding hygiene practices and a healthy attitude toward menstruation ${ }^{17}$. Thus, school absenteeism was high among adolescent girls with illiterate mothers. Adolescent girls who come from a nuclear family and had no knowledge about menstrual hygiene management before menarche were more absent from school. A study conducted in Dibrugarh town ${ }^{1}$ reported that adolescent girls from single/nuclear families were more absent from school during menstruation. It also found that girls from an extended family had good knowledge of menstrual hygiene management, as found in previous studies ${ }^{18,19}$. School absenteeism among the adolescent girls was lower for those who received information about menstrual hygiene management from school-based programs, as found in a previous study ${ }^{20}$. Hence, this study suggests that school-based menstrual hygiene practice intervention programs should be increased to reduce school absenteeism among school-going adolescent girls.

The present study revealed that poor menstrual hygiene practices were significantly associated with absenteeism among school-going adolescent girls during menstrual 
periods, in line with previous studies ${ }^{4,21}$. A study conducted in India reported that poor hygiene management was associated with reproductive tract infections ${ }^{21}$. In our study poor hygiene management was considered if: the frequency of change of sanitary pad/cloth was greater than eight hours; not cleaning hands before and after changing MHM materials; not washing external genitalia at least once per day; and using reusable sanitary pads/cloths without washing them with soap/antiseptic. This type of poor hygiene is intricately linked with risks of infection, such as urogenital infections, yeast infection, fungal infection, and urinary tract infection $^{21-23}$, leading to increased school absenteeism during menstrual periods.

This study found that school absenteeism was higher among adolescent girls who used homemade cloths or underwear, reused sanitary materials, washed reusable cloths with only water and dried these inside the house or outside without sunlight. School absenteeism was less among the girls who use sanitary pads during this period. School absenteeism was significantly associated with the reuse of sanitary materials, consistent with results found with a study conducted in Northeast Ethiopia ${ }^{3}$. The adolescent girls who used sanitary pads were more concern about menstruation and hygiene practices ${ }^{17}$. Due to proper hygiene maintenance and concern about menstruation, they had less reproductive tract infections and less school absenteeism. Reusable sanitary cloth washing with soap or antiseptic results in properly disinfected or less contaminated cloths that inhibit several genitival problems and less infection ${ }^{24}$. Improper drying of reusable cloths may be associated with contamination and responsible for reproductive tract infections found in a previous study ${ }^{25}$. Unsatisfactory cleaning or cleaning without soap/antiseptics also leads to less school participation by adolescent girls during menstrual periods. This issue is responsible for reproductive infection $\mathrm{s}^{26,27}$ and pain during menstruation ${ }^{28}$.

\section{Strengths and limitations}

This study has some strengths. It is one of the first studies to explore school absenteeism among school-going adolescent girls in Bangladesh and assesses some of the factors associated with school absenteeism. However, this study has limitations. School absenteeism was considered a full-day absence from school, but evidence suggests that girls often miss only part of the school day. School wash facilities of the study participants were not visited, and household wash facility data were not collected. This study suggests further longitudinal research to interpret the association between school wash facilities, poor menstrual hygiene management, and poor wash facilities at home with school absenteeism.

\section{CONCLUSIONS}

The major finding of this study was that the lack of knowledge and the lack of wash facilities and soap/ antiseptic equipment for washing sanitary pads, for bathing and cleaning external genitalia were responsible for school absenteeism among school-going adolescent girls. Menstrual hygiene management knowledge and adequate mother's education level prevent school absenteeism. This study's findings may help governmental and nongovernmental organizations to design programs to reduce school absenteeism during menstrual periods by improving education on health hygiene practices during the menstrual cycle.

\section{REFERENCES}

1. Sonowal P, Talukdar K. Menstrual Hygiene Knowledge and Practices amongst Adolescent Girls in Urban Slums of Dibrugarh Town- A Cross Sectional Study. Galore International Journal of Health Sciences and Research. 2019;4(1):44-51. Accessed October 23, 2020. https://www. gijhsr.com/GIJHSR_Vol.4_Issue.1_Jan2019/9.pdf

2. Sumpter C, Torondel B. A systematic review of the health and social effects of menstrual hygiene management. PLoS One. 2013;8(4):e62004. doi:10.1371/journal.pone.0062004

3. Tegegne TK, Sisay MM. Menstrual hygiene management and school absenteeism among female adolescent students in Northeast Ethiopia. BMC Public Health. 2014;14:1118. doi:10.1186/1471-2458-14-1118

4. Davis J, Macintyre A, Odagiri M, et al. Menstrual hygiene management and school absenteeism among adolescent students in Indonesia: evidence from a crosssectional school-based survey. Trop Med Int Health. 2018;23(12):1350-1363. doi:10.1111/tmi.13159

5. UNICEF. Solomon Islands: Incorporating MHM into national WASH in schools policies and guidelines. Presented at: 4 th MHM Virtual Conference, 22-23 October 2015. Accessed October 23, 2020. http://docplayer.net/39281011-Solomonislands-incorporating-mhm-into-national-wash-in-schoolspolicies-and-guidelines.html

6. SNV Netherlands Development Organisation. Girls in Control: Compiled Findings from Studies on Menstrual Hygiene Management of Schoolgirls. Published 2015. Accessed October 23, 2020. https://snv.org/cms/sites/default/files/ explore/download/snv_girls_in_control_baseline_report.pdf

7. Patton GC, Sawyer SM, Santelli JS, et al. Our future: a Lancet commission on adolescent health and wellbeing. Lancet. 2016;387(10036):2423-2478. doi:10.1016/S0140-6736(16)00579-1

8. Anee US, Islam A, Wahid F, Ahmed MK. Knowledge, Attitude and Practice on Female Hygiene Management among Madrasa Girls in Dhaka, Bangladesh. Asian Journal of Multidisciplinary Studies. 2020;3(1):110-116.

9. National Institute of Population Research and Training (NIPORT), Mitra and Associates, ICF International. Bangladesh Demographic and Health Survey 2014. Published March 2016. Accessed October 23, 2020. https:// dhsprogram.com/pubs/pdf/FR311/FR311.pdf

10. Snehalatha M, Fonseca C, Rahman M, Uddin R, Ahmed M, Joy Sharif A. School WASH programmes in Bangaldesh: how 
much does it cost? - Applying the life-cycle costs approach in selected upazilas. Published March 2015. Accessed October 23, 2020. https://www.ircwash.org/sites/default/files/ lcca_methodologyreport_school_wash_clean.pdf

11. International Centre for Diarrheal Diseases Research, Bangladesh (icddr,b), WaterAid Bangladesh, Policy Support Unit (PSU). Bangladesh National Hygiene Baseline Survey: Preliminary Report. Published June 2014. https:// washmatters.wateraid.org/publications/bangladeshnational-hygiene-baseline-survey-preliminary-report-2014

12. Vashisht A, Pathak R, Agarwalla R, Patavegar BN, Panda M. School absenteeism during menstruation amongst adolescent girls in Delhi, India. J Family Community Med. 2018;25(3):163-168. doi:10.4103/jfcm.JFCM_161_17

13. House S, Mahon T, Cavill S. Menstrual hygiene matters: A resource for improving menstrual hygiene around the world. Published 2012. Accessed October 23, 2020. https:// washmatters.wateraid.org/sites/g/files/jkxoof256/files/ Menstrual\%20hygiene\%20matters\%20low\%20resolution. pdf

14. Oster E, Thornton R. Menstruation, Sanitary Products, and School Attendance: Evidence from a Randomized Evaluation. Am Econ J Appl Econ. 2011;3(1):91-100. doi:10.1257/app.3.1.91

15. Miiro G, Rutakumwa R, Nakiyingi-Miiro J, et al. Menstrual health and school absenteeism among adolescent girls in Uganda (MENISCUS): a feasibility study. BMC Womens Health. 2018;18(1):4. doi:10.1186/s12905-017-0502-z

16. Aniebue UU, Aniebue PN, Nwankwo TO. The impact of pre-menarcheal training on menstrual practices and hygiene of Nigerian school girls. Pan Afr Med J. 2009;2:9. PMID:21532905.

17. Thakre SB, Thakre SS, Reddy M, Rathi N, Pathak K, Ughade S. Menstrual Hygiene: Knowledge and Practice among Adolescent School Girls of Saoner, Nagpur District. J Clin Diagn Res. 2011;5(5):1027-1033. Accessed October 23, 2020. https://www.jcdr.net/articles/PDF/1522/30\%20-\%202941. pdf

18. Asha AC, Karim NB, Bakhtiar M, Rahaman KS. Adolescent athlete's knowledge, attitude and practices about menstrual hygiene management (MHM) in BKSP, Bangladesh. Asian Journal of Medical and Biological Research. 2019;5(2):126137. doi:10.3329/ajmbr.v5i2.42494

19. Hennegan J, Nansubuga A, Smith C, Redshaw M, Akullo A, Schwab KJ. Measuring menstrual hygiene experience: development and validation of the Menstrual Practice Needs Scale (MPNS-36) in Soroti, Uganda. BMJ Open. 2020;10(2):e034461. doi:10.1136/bmjopen-2019-034461

20. Haque SE, Rahman M, Itsuko K, Mutahara M, Sakisaka K.

CONFLICTS OF INTEREST

The authors have completed and submitted the ICMJE Form for Disclosure of Potential Conflicts of Interest and none was reported.
The effect of a school-based educational intervention on menstrual health: an intervention study among adolescent girls in Bangladesh. BMJ Open. 2014;4(7):e004607. doi:10.1136/bmjopen-2013-004607

21. Torondel B, Sinha S, Mohanty JR, et al. Association between unhygienic menstrual management practices and prevalence of lower reproductive tract infections: a hospital-based cross-sectional study in Odisha, India. BMC Infect Dis. 2018;18(1):473. doi:10.1186/s12879-018-3384-2

22. Ali TS, Sami N, Khuwaja AK. Are unhygienic practices during the menstrual, partum and postpartum periods risk factors for secondary infertility? J Health Popul Nutr. 2007;25(2):189-194. PMID:17985820.

23. Hennegan J, Shannon AK, Rubli J, Schwab KJ, Melendez-Torres GJ. Women's and girls' experiences of menstruation in lowand middle-income countries: A systematic review and qualitative metasynthesis. PLoS Med. 2019;16(5):e1002803. doi:10.1371/journal.pmed.1002803

24. Das P, Baker KK, Dutta A, et al. Menstrual Hygiene Practices, WASH Access and the Risk of Urogenital Infection in Women from Odisha, India. PLoS One. 2015;10(6):e0130777. doi:10.1371/journal.pone.0130777

25. Sivakami M, Maria van Eijk A, Thakur H, et al. Effect of menstruation on girls and their schooling, and facilitators of menstrual hygiene management in schools: surveys in government schools in three states in India, 2015. J Glob Health. 2019;9(1):010408. doi:10.7189/jogh.09.010408

26. Khanna A, Goyal RS, Bhawsar R. Menstrual Practices and Reproductive Problems: A Study of Adolescent Girls in Rajasthan. J Health Manag. 2005;7(1):91-107. doi:10.1177/097206340400700103

27. Su JJ, Lindell D. Promoting the menstrual health of adolescent girls in China. Nurs Health Sci. 2016;18(4):481-487. doi:10.1111/nhs.12295

28.Zegeye DT, Megabiaw B, Mulu A. Age at menarche and the menstrual pattern of secondary school adolescents in northwest Ethiopia. BMC Womens Health. 2009;9:29. doi:10.1186/1472-6874-9-29

PROVENANCE AND PEER REVIEW

Not commissioned; externally peer reviewed.

FUNDING

There was no source of funding for this research. 